\title{
Phase structure of Topologically massive gauge theory with fermion
}

\author{
Yuichi Hoshino \\ Kushiro National College of Technology, \\ Otanoshike-nishi 2-32-1,Kushro 084-0916,Hokkaido,Japan
}

April 21, 2022

\begin{abstract}
Using Bloch-Nordsieck approximation fermion propagator in 3-dimensional gauge theory with topological mass is studied.Infrared divergence of ChernSimon term is soft,which modifes anomalous dimension.In unquenched QCD with 2-component spinor anomalous dimension has fractional value, where order parmeter is divergent.
\end{abstract}

\section{Introduction}

The Lagrangeans of Topologically massive gauge theory with fermion are(1)

$$
\begin{gathered}
L=\frac{1}{4} F_{\mu \nu} F^{\mu \nu}+\frac{1}{4} \theta \epsilon^{\mu \nu \rho} F_{\mu \nu} A_{\rho}+\bar{\psi}(i \gamma \cdot(\partial-i e A)-m) \psi+\frac{1}{2 d}(\partial \cdot A)^{2} \\
L=\frac{1}{4 g^{2}} \operatorname{tr}\left(F_{\mu \nu} F^{\mu \nu}\right)-\frac{\theta}{4 g^{2}} \epsilon^{\mu \nu \rho} \operatorname{tr}\left(F_{\mu \nu} A_{\rho}-\frac{2}{3} A_{\mu} A_{\nu} A \rho\right) \\
+\bar{\psi}(i \gamma \cdot(\partial-i e A)-m) \psi+\frac{1}{2 d}(\partial \cdot A)^{2}
\end{gathered}
$$

,where $\theta=\left(g^{2} / 4 \pi\right) n,(n=0, \pm 1, \pm 2 \ldots)$ with $\gamma$ matrix for 4-comonent fermion (2).In comparison with massless $Q E D_{3}, Q C D_{3}$ topological mass term seems to soften the infrared divergence of massive fermion near its on-shell.In Minkowski metric we have $5 \gamma$ matrices $\left\{\gamma_{\mu}, \gamma_{\nu}\right\}=2 g_{\mu \nu},(\mu=0,1,2)$

$$
\begin{aligned}
\gamma_{0} & =\left(\begin{array}{cc}
\sigma_{3} & 0 \\
0 & -\sigma_{3}
\end{array}\right), \gamma_{1,2}=-i\left(\begin{array}{cc}
\sigma_{1,2} & 0 \\
0 & -\sigma_{1,2}
\end{array}\right), \gamma_{4}=\left(\begin{array}{cc}
0 & I \\
I & 0
\end{array}\right), \gamma_{5}=\left(\begin{array}{cc}
0 & -i I \\
i I & 0
\end{array}\right) \\
\tau & \equiv \frac{-i}{2}\left[\gamma_{4}, \gamma_{5}\right]=\operatorname{diag}(I,-I), \tau_{ \pm}=\frac{1 \pm \tau}{2}
\end{aligned}
$$

There are two redundant matrices $\gamma_{4}$ and $\gamma_{5}$ which anticommutes with other three $\gamma$ matrices. There exists two kind of chiral transformation $\psi \rightarrow \exp \left(i \alpha \gamma_{4}\right) \psi, \psi \rightarrow$ 
$\exp \left(\alpha \gamma_{5}\right) \psi$. The matrices $\left\{\gamma_{4}, \gamma_{5}, I_{4}, \tau\right\}$ generate a $U(2)$ chiral symmetry containing massless spinor fields. This $U(2)$ symmetry is broken down to $U(1) \times U(1)$ by a spinor mass term $m_{e} \bar{\psi} \psi$ and parity violating mass $m_{o} \bar{\psi} \tau \psi$, where $\bar{\psi} \tau \psi$ is a spin density.Here after we take 4-component spinors to study chiral symmetry breaking by $m^{e} \bar{\psi} \psi$ in pure $\mathrm{QED}_{3}$, where $\bar{\psi} \tau \psi$ is invariant under chiral transformation.After that the effects of Chern-Simon term will be studied by 2-component spinors.

\section{Fermion spectral function}

The spectral function of 4-component fermion and photon propagator(3) are defined as

$$
\begin{gathered}
S_{F}(x)=S_{F}^{0}(x) \exp (F(x)), S_{F}^{0}(x)=-(i \gamma \cdot \partial+m) \frac{\exp \left(-m \sqrt{-x^{2}}\right)}{4 \pi \sqrt{x^{2}}} . \\
D_{F}^{0}(k)=-i\left(\frac{g_{\mu \nu}-k_{\mu} k_{\nu} / k^{2}-i \theta \epsilon_{\mu \nu \rho} k^{\rho} / k^{2}}{k^{2}-\theta^{2}+i \epsilon}\right)+i d \frac{k_{\mu} k_{\nu}}{k^{4}},
\end{gathered}
$$

where $F$ is an $O\left(e^{2}\right)$ matrix element $\left|T_{1}\right|^{2}$ for the process electron $(p+k) \rightarrow$ electron $(p)+$ photon $(k)$ as

$$
\begin{gathered}
T_{1}=-i e \frac{\epsilon_{\mu}(k, \lambda)}{\gamma \cdot(p+k)-m} \gamma^{\mu} \exp (i(p+k) \cdot x) U(p, s), \\
\sum_{\lambda, S} T_{1} \overline{T_{1}}=-\frac{\gamma \cdot p+m}{2 m} e^{2}\left[\frac{m^{2}}{(p \cdot k)^{2}}+\frac{1}{p \cdot k}+\frac{(d-1)}{k^{2}}\right]-\frac{\gamma \cdot p}{m} \frac{e^{2}}{4 \theta} \frac{m}{p \cdot k}, \\
F=\int \frac{d^{3} k}{(2 \pi)^{2}} \exp (i k x) \theta\left(k_{0}\right) \delta\left(k^{2}-\theta^{2}\right) \sum_{\lambda, S} T_{1} \overline{T_{1}} .
\end{gathered}
$$

Here we use the retarded propagator to derive the function $F$

$$
D_{+}(x)=\int \frac{d^{3} k}{i(2 \pi)^{2}} \exp (i k \cdot x) \theta\left(k_{0}\right) \delta\left(k^{2}-\theta^{2}\right)=\frac{\exp \left(-\theta \sqrt{-x^{2}}\right)}{8 \pi i \sqrt{-x^{2}}}
$$

The function $F$ is evaluated by $\alpha$ integration for pure $\mathrm{QED}_{3}(4,3)$.

$$
\begin{aligned}
F & =i e^{2} m^{2} \int_{0}^{\infty} \alpha d \alpha D_{F}(x+\alpha p)-e^{2} \int_{0}^{\infty} d \alpha D_{F}(x+\alpha p)-i(d-1) e^{2} \frac{\partial}{\partial \theta^{2}} D_{F}\left(x, \theta^{2}\right) \\
& =\frac{e^{2}}{8 \pi}\left[\frac{\exp (-\theta|x|)-\theta|x| E_{1}(\theta|x|)}{\theta}-\frac{E_{1}(\theta|x|)}{m}+\frac{(d-1) \exp (-\theta|x|))}{2 \theta}\right] .
\end{aligned}
$$

It is well known that function $F$ has linear and logarithmic infrared divergence with respect to $\theta$ where $\theta$ is a bare photon mass.Here we notice the followings.(1) $\exp (F)$ includes all infrared divergences.(2) quenhed propagator has linear and logarithmic infrared divergences.Linear divegence is absent in a special gauge.In unquenched case $\theta$ dependence of $\exp (F)$ is modified by dressed boson spectral function to $\int d s \rho_{\gamma}(s) \exp \left(F(x, \sqrt{s})\right.$, where $\pi \rho_{\gamma}(s)=-\Im(s-\Pi(s))^{-1}$. 


\section{Phase strucutures}

For short and long distance we have the approximate form of the function $F$

$$
\left.F_{S} \sim A-\theta|x|+(D+C|x|) \ln (\theta|x|)\right)-\frac{(d+1-2 \gamma) e^{2}|x|}{16 \pi},(\theta|x| \ll 1), F_{L} \sim 0,(1 \ll \theta|x|) . .
$$

From the above formulae we have

$$
\begin{aligned}
\exp (F) & =A(\theta|x|)^{D+C|x|}(\theta|x| \ll 1), A=\exp \left(\frac{e^{2}(1+d)}{16 \pi \theta}+\frac{e^{2} \gamma}{8 \pi m}\right), \\
C & =\frac{e^{2}}{8 \pi}, D=\frac{e^{2}}{8 \pi m},
\end{aligned}
$$

where $m$ is the physical mass and $\gamma$ is an Euler's constant.Here we see $D$ acts to change the power of $|x|$. For $D=1, S_{F}(0)$ is finite and we have $\langle\bar{\psi} \psi\rangle \neq 0$ (4) 6). Thus if we require $D=1$, we obtain $m=e^{2} / 8 \pi$.In the same way we add the conrtribution of Chern-Simon term.For simplicity we consider the 2-component spinors in(7).In the condensed phase we have the modified anomalous dimension for $\theta>0$

$$
D=\frac{e^{2}}{8 \pi m}+\frac{e^{2}}{32 \pi \theta}=1, m^{\prime}=\frac{e^{2}}{8 \pi} /\left(1-\frac{e^{2}}{32 \pi \theta}\right), \Delta m=\frac{e^{2}}{8 \pi} \frac{e^{2}}{32 \pi \theta} /\left(1-\frac{e^{2}}{32 \pi \theta}\right) .
$$

In unquenched case there are parity even and odd spectral function of gauge boson by vacuum polarization(1).In this case we separate $D$ into parity even and odd contribution $D^{e}=e^{2} / 8 \pi m, D^{O}=e^{2} / 32 \pi \theta$. For Topologically Massive $\mathrm{QCD}, \theta$ is quantized with $n(0, \pm 1, \pm 2, .$.$) . Thus we have D=e^{2} /(8 \pi m)+1 / 8 n=$ 1 for quenched case, and $D^{e}=e^{2} / 8 \pi m=1, D^{O}=1 /(8 n)$ for unquenched

case,which leads to $\langle\bar{\psi} \psi\rangle=\infty$ for any $n \neq 0$.In the 4 -component spinor free fermion propagator is decomposed into chiral representation

$$
S_{F}(p)=\frac{1}{m_{\epsilon} I+m_{O} \tau-\gamma \cdot p}=\frac{\left(\gamma \cdot p+m_{+}\right) \tau_{+}}{p^{2}-m_{+}^{2}+i \epsilon}+\frac{\left(\gamma \cdot p+m_{-}\right) \tau_{-}}{p^{2}-m_{-}^{2}+i \epsilon} .
$$

The difference in two spectral functions is a opposite sign of each Chern-Simon contribution for $\tau_{ \pm}$.In Toplogically massive gauge theory dynamical mass is parity even and Chern-Simon term shifts mass of different chirality with opposite sign.However shifted mass may not strongly depend on $\theta$ but $\langle\bar{\psi} \psi\rangle$ is proportional to $\theta(12)(\sqrt[5]{)})$. Our approximation is convenient for unquenched case by the use of gauge boson spectral function( $(6)$.

\section{References}

[1] S.Deser,R.Jackiw\&Templeton,Annals of Physics 281,409-449(2000).

[2] C.J.Burden,NuclerPhysicsB387(1992)419-446,Kei-ichi Kondo,Int.J.Mod.Phys.A11;777-822,1996. 
[3] R.Jackiw,L.Soloviev,Phys.Rev.173.5(1968)1485.

[4] Yuichi Hoshino,in CONTINUOUS ADVANCES IN QCD 2008;361-372.

[5] Toyoki Matsuyama,Hideko Nagahiro,Mod.Phys.Lett.A15 (2000) 2373-2386.

[6] C.S.Fisher,Reinhart Alkofer,T.Darm,P.Maris,Phys.Rev.D70:073007,2004. 